Journal of Engineering and Applied Sciences 14 (6): 1922-1927, 2019

ISSN: 1816-949X

(C) Medwell Journals, 2019

\title{
Simulation-Based Comparative Study of Routing Protocols for Wired Networks
}

\author{
Belal Ayyoub, Bilal Zahran, Jihad Nader, Jamil al Al-Azzah and Ahmed Sharadqeh \\ Department of Computer and Network Engineering, Faculty of Engineering Technology, \\ Al-Balqa Applied University, Amman, Jordan
}

\begin{abstract}
Wired networks have gained enormous concern from researchers and application developers because of the widely used of their network type. Dynamic routing is the process of finding the best path from source to destination to deliver data packets in the network. Routing paly a significant rule of the overall performance of the wired network. Each routing protocol has an algorithm and metrics that vary in determining the optimal path to a network. Therefore, the developers developed several routing protocols to address all aspects of routing process. The aim of this study is to compare the performance of four routing protocols namely; RIPv2, OSPF, IGRP and EIGRP experimentally. To generalize the results obtained we tested the performance of the routing protocols utilizing different application and two different scenarios. The applications used in the experiments are e-mail, http and database. The simulation was performed using OPNET. The results showed that in general EIGRP protocol outperform others. Furthermore, specific protocols behave well in some scenarios.
\end{abstract}

Key words: Dynamic Routing Protocols (RP), convergence time, RIPv2, OSPF, EIGRP, IGRP

\section{INTRODUCTION}

The purpose of dynamic routing protocols is to direct data traffic down the optimal path toward a destination when multiple paths are available at the same time. The "dynamic" part refers to changing of routing tables if the network topology changes. Every dynamic routing protocol is associated with an algorithm and metrics to determine the optimal path. Here, we examined four most popular routing protocols used within enterprises.

Routing Information Protocol version 2 (RIPv2) is a distance-vector routing protocols which employ the hop count as a routing metric. RIPv2 suffered from scalability issues due to a relatively low maximum hop count of 15 routing devices (Fitzgerald et al., 2017; Clark, 2003). Interior Gateway Routing Protocol (IGRP) is an advanced distance vector protocol. But in terms of scalability and convergence times, IGRP significantly outperform RIPv2. IGRP supports four metrics for each route, including bandwidth, delay, load and reliability. IGRP is considered a classful routing protocol. Cisco created EIGRP (Enhanced Interior Gateway Routing Protocol) to enhance the addressing issues and other parameters of IGRP. EIGRP adds support for Variable Length Subnet Mask (VLSM) and adds the Diffusing Update Algorithm (DUAL) in order to improve routing and provide a loopless environment. EIGRP has completely replaced
IGRP, making IGRP an obsolete routing protocol. We examined IGRP here for experimental purposes only. EIGRP was Cisco proprietary routing protocol that only ran on Cisco hardware and software but in 2013, Cisco opened EIGRP up as an IETF draft (Pethe and Burnase, 2011; Sharma et al., 2012). Open Shortest Path First (OSPF) is a Link State Routing (LSR) protocol. OSPF is a widely used Interior Gateway Protocol (IGP) in large enterprise networks. It implements the Dijkstra's algorithm, also known as Shortest Path First (SPF) algorithm. The shortest path through a network was calculated based on the cost of the route, taking into account the bandwidth (Fitzgerald et al., 2017; Clark, 2003).

Literature review: Routing in wired networks gained a massive concern form researchers. Several comparison studies are conducted to test and compare the performance of several protocols (Mohammad et al., 2017; Wandile et al., 2017; Verma and Bhardwaj, 2016; Singh and Mahajan, 2013; Vetriselvan et al., 2014; Meenakshi et al., 2014; Krinpayorm and Pattaramalai, 2012).

Mohammad et al. (2017), the researchers studied routing in enterprise networks that use multiple routing protocols. They studied three scenarios with diverse routing protocols configuration on the same network.

Corresponding Author: Belal Ayyoub, Department of Computer and Network Engineering, Faculty of Engineering Technology, Al-Balqa Applied University, Amman, Jordan 
The simulation showed the performance of the EIGRP-IGRP-ISIS scenario that is better in terms of FTP download response time, video packet delay variation, throughput and network convergence time as compared with the other scenarios in this study. On the other hand, the performance of the OSPF-IGRP-ISIS scenario outperforms the two other scenarios in terms of voice packet delay variation, voice and video conferencing packet end to end delay and queuing delay. In contrast, the OSPF-IGRP-EIGRP scenario is better than the others in terms of FTP upload response time and voice jitter. By Wandile et al. (2017), the researchers studied the performance of four major types of routing protocols; RIPv2, OSPF, EIGRP and IS-IS using riverbed modeler. Tree and Mesh topologies had been built and the simulation of each routing protocols in all three topologies had been performed. At first implemented the three routing protocols into a small tree network and evaluated the convergence activity, convergence duration and traffic sent (bytes/sec) to compare the difference in their performance and after that they implemented the three protocols into large mesh and large tree topologies and evaluated the same three parameters. IS-IS is the fastest among all four. OSPF has a longer initialization time compare to RIPv2, EIGRP and IS-IS. Both OSPF and RIPv2 have longer initialization time as compared to EIGRP and IS-IS. EIGRP has longer initialization time than IS-IS.

Verma and Bhardwaj (2016), the researchers compared between the RIPv 2 and OSPF protocol from reviewing studies conducted earlier. OSPF outperforms the RIPv 2 in term various efficient throughput and packet delay in networks. But the RIPv2 is enhanced by using the triggered-RIPv2, OSPF adjust the link and coverage better than RIPv2. RIPv2 offers better performance than OSPF in a limited number of nodes.

\section{MATERIALS AND METHODS}

In general, the operations of a dynamic routing protocol can be described as follows:

- The router sends and receives routing messages on its interfaces

- The router shares routing messages and routing information with other routers that are using the same routing protocol

- Routers exchange routing information to learn about remote networks
- When a router detects a topology change the routing protocol can advertise this change to other routers.

One of the most parameters of comparing routing protocols is the convergence time. The convergence time is the time it takes routers to share information, calculate best paths and update their routing tables. The contribution of our research is to assess the performance of the four routing protocols utilizing different network applications namely; database application, HTTP and e-mail. The performance of each routing protocol is computed for each specific application. Here are the descriptions of these applications.

The default transport protocol used in the e-mail application provided with the Software is TCP, i.e., messages are sent and received using TCP. Modern e-mail packages use a combination of SMTP (Simple Mail Transfer Protocol) and POP (Post Office Protocol). Both SMTP and POP use TCP as the underlying transport. SMTP transfers an e-mail from the client to the mail server.

A database application enables the user to store information. Database operations are divided into two categories; a database entry and a database query.

A database entry results in a fixed amount of data being written into the database and a database query results in the client issuing a query and the server responding with some data. The default transport protocol for the database application is TCP.

The HTTP application models Web browsing. The user downloads a page from a remote server. The page contains text and graphic information (also referred to as "inlineobjects") study

\section{RESULTS AND DISCUSSION}

Experimental setup: We sat up two main scenarios of networks under which several scenarios are branched. The first main scenario is to compute performance measures without link failure while the other with link failure. The aim of scenario with link failure is to compute convergence time of routing protocols. Each scenario uses a specific dynamic routing protocol; RIPv2, OSPF, IGRP or EIGRP. We investigated the behavior of the routing protocols under three different applications: database, e-mail and http. Each service of the three applications is hosted by its own server. We used OPNET simulator to set up the scenarios and to calculate the performance metrics. Figure 1 an example of a network topology built using OPNET simulator. 


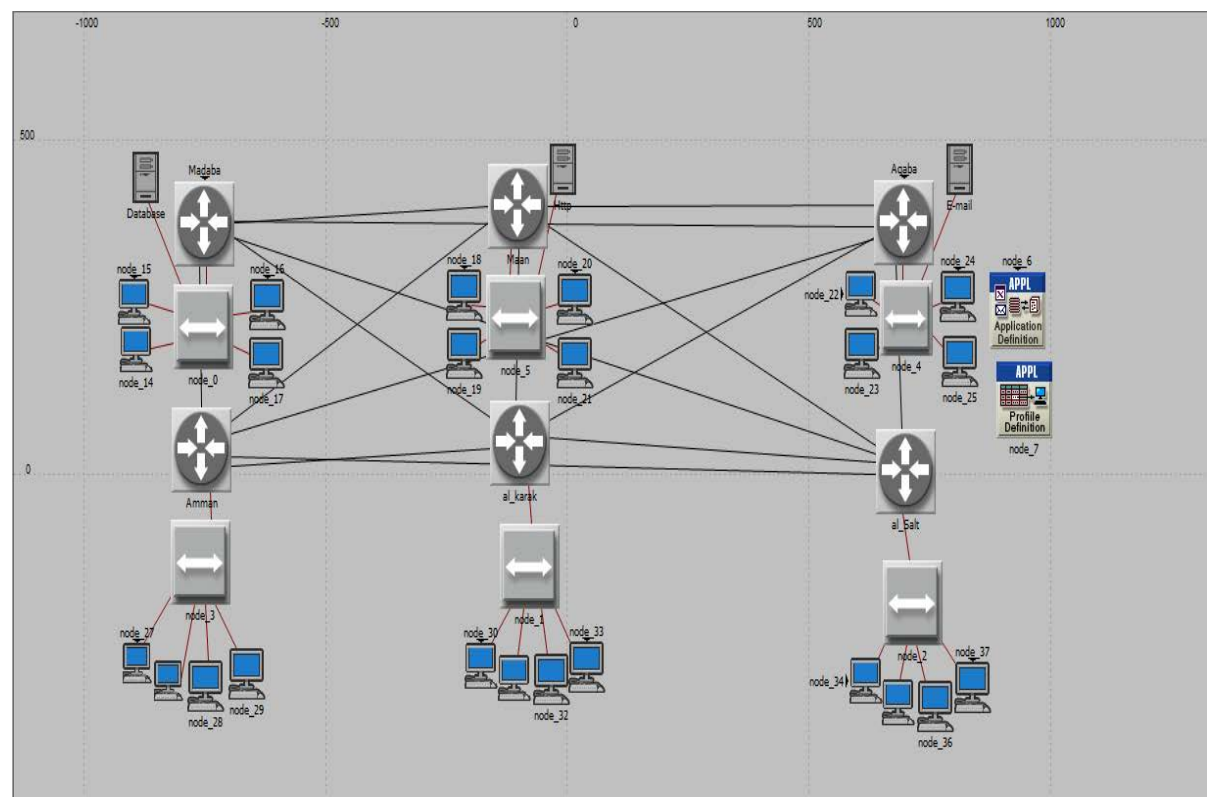

Fig. 1: An example of a network topology

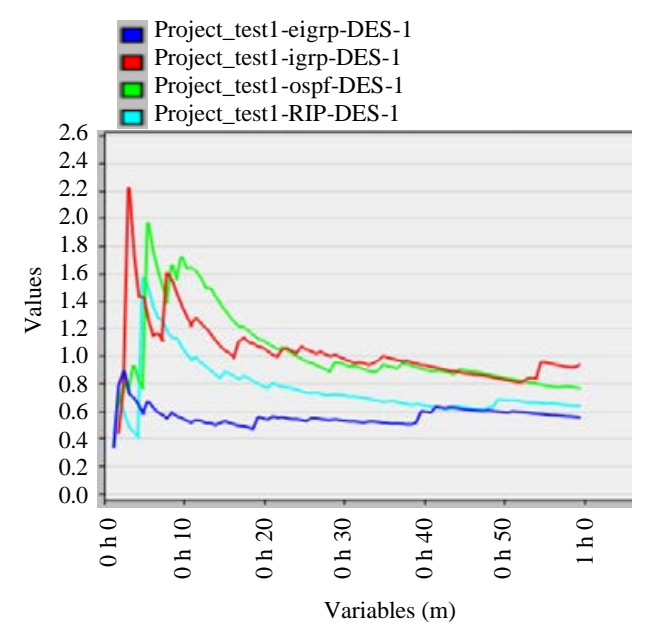

Fig. 2: Database query response time (sec) for $4 \mathrm{RPs}$; Time-average (in DB query response time $(\mathrm{sec})$ )

Performance of RPs: We defined a performance metrics for the comparison purpose as follows; for database application, the metrics are query response time, data rate of traffic sent and received. For e-mail application, the metrics are download time, upload time, data rate of traffic sent and received. For HTTP application, the metrics are page response time, object response time, data rate of traffic sent and received.

Database application: Figure 2 shows the query response time for four routing protocols. The EIGRP protocols took the minimum average time among the four protocols. Furthermore, RIPv2 behavior is accepted. Figure 3 shows the performance of data rate of database traffic (sent and received) measured in bytes/second.

All RPs behaviors are close to each other while dealing with the data rate of traffic sent and received with database application. OSPF has slightly better performance than others.

Application: E-mail, RIPv2 and EIGRP recorded the minimum time values while IGRP recorded the highest time values when dealing with e-mail application (download/upload). Figure 4 shows the e-mail download and upload response time.

Application HTTP: Figure 5 shows the page and object response time when dealing with http application. OSPF performs the best in speed than other RPs while the RIPv2 is the slowest RP.

Figure 6 shows the data rate of traffic sent and received while dealing with http RIPv 2 records the highest time values (worst) in HTTP traffic sent and received and EIGRP records the lowest value.

Summary of results: Table 1 and 2 show the results of the performance of the four routing protocols under normal traffic and with link failure. Performance given numbers starting from 1 , the best and last number 4 which indicate the worst performance. 

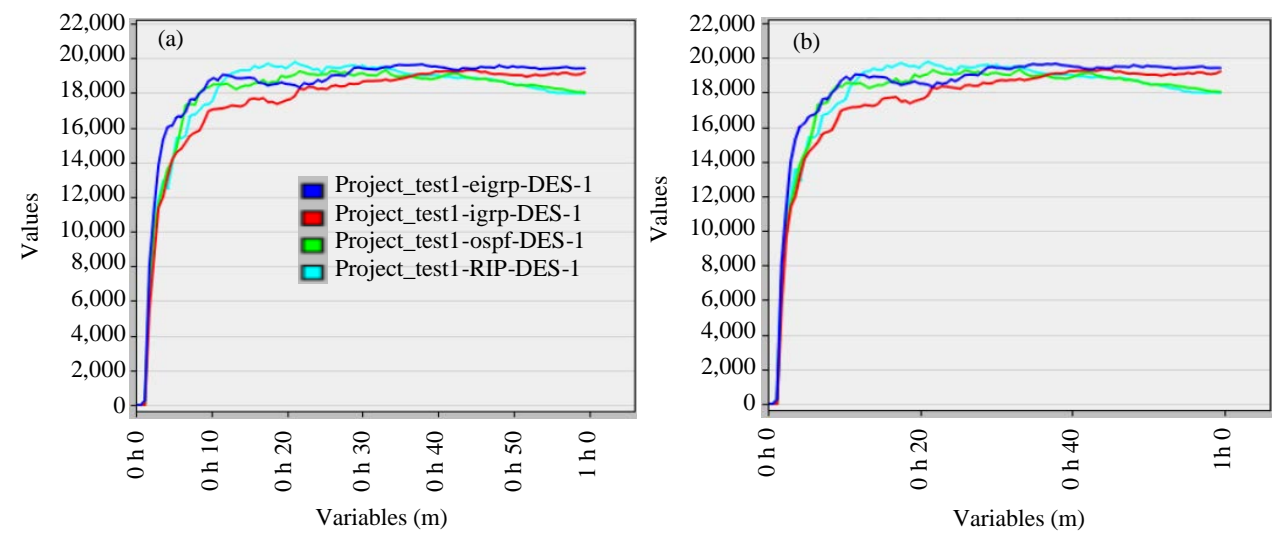

Fig. 3: Data rate for query traffic, received and sent: a) Time-average (in $\mathrm{dB}$ query. Traffic received (bytes/sec)) and b) Time-average (in $\mathrm{dB}$ query. Traffic sent (bytes/sec))
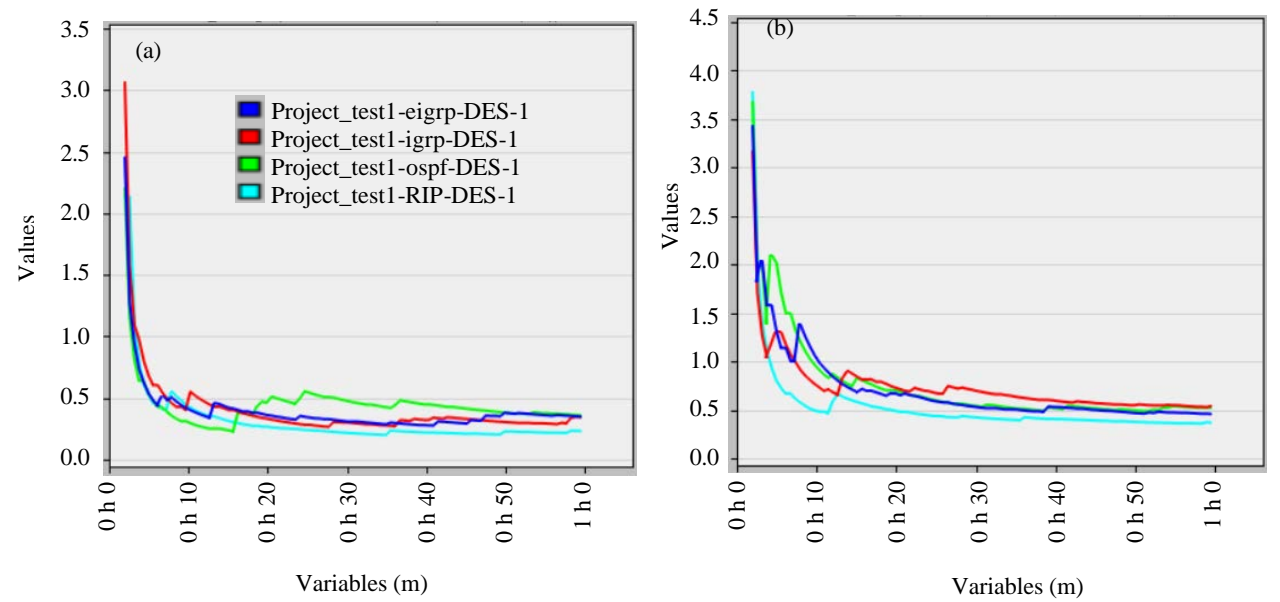

Fig. 4: E-mail download and upload times: a) Time-average (in e-mail. Download response time (sec)) and b) Time-average (in e-mail. Download response time (sec))
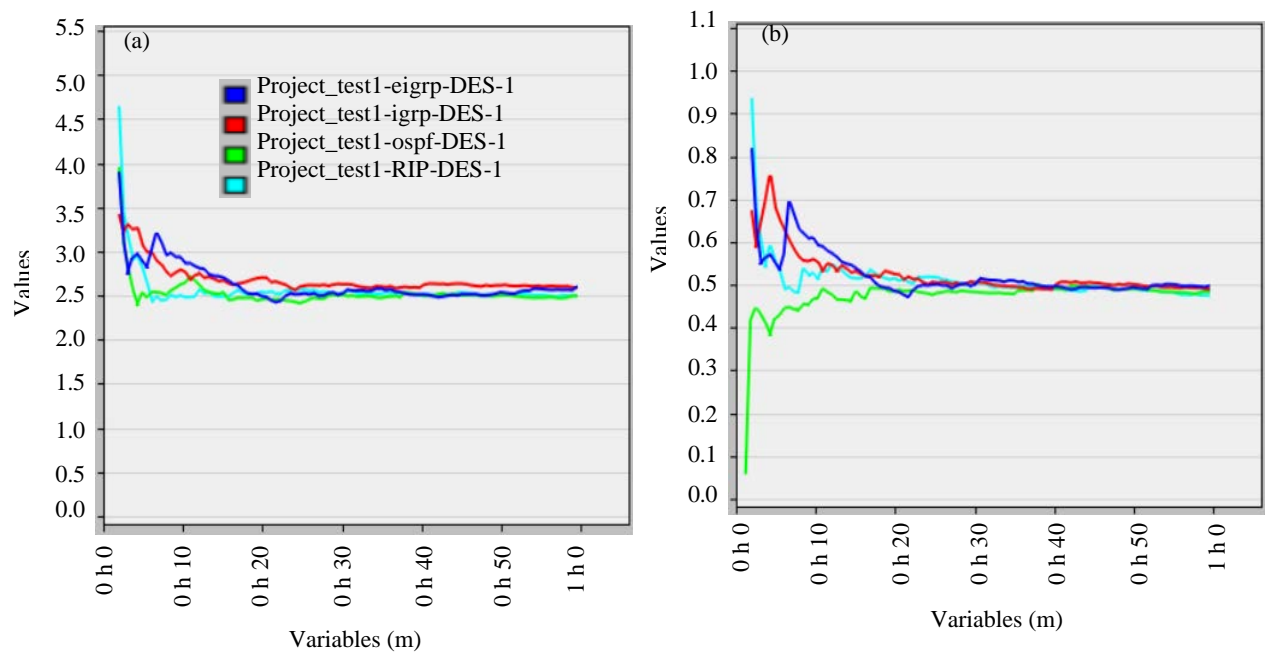

Fig. 5: HTTP Page and object response time: a) Time-average (in HTTP. Page response time (sec)) and b) Time-average (in HTTP. Object response time (sec)) 

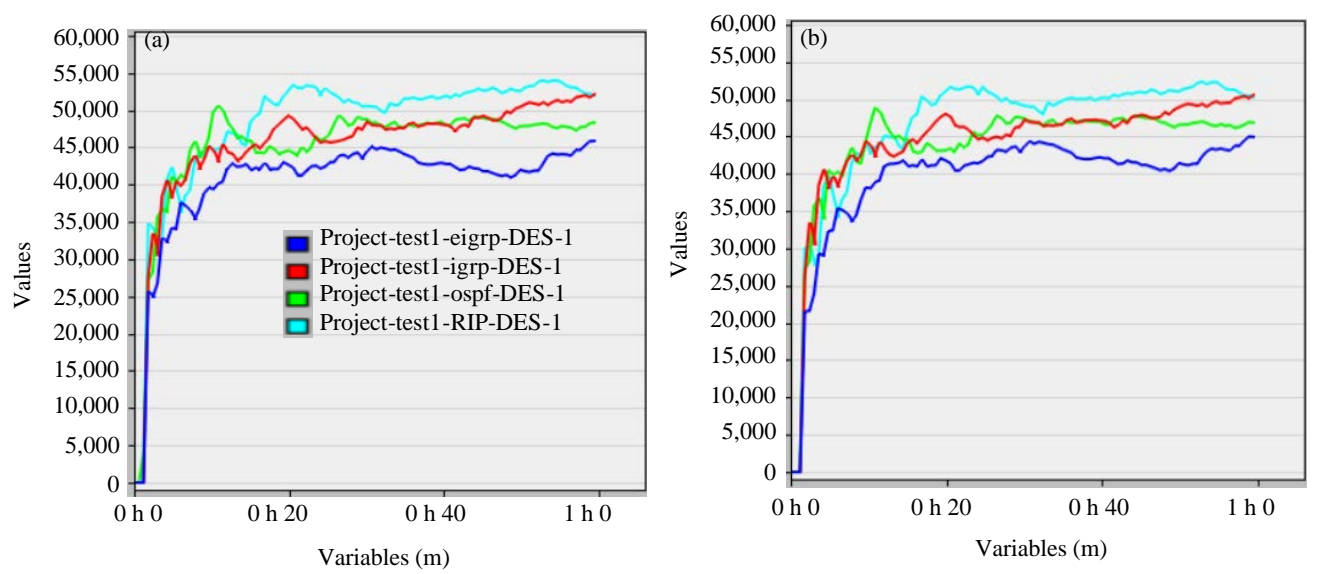

Fig. 6: HTTP page response time: a) Time-average (in HTTP. Traffic sent (bytes/sec)) and b) Time-average (in dB query. Traffic received (bytes/sec))

Table 1: Performance of RPs with normal traffic

\begin{tabular}{llllc}
\hline & & \multicolumn{3}{c}{ HTTP } \\
RP/Application & e-mail & $\mathrm{dB}$ & Page response & Traffic \\
\hline RIPv2 & 2 & 2 & 3 & 3 \\
IGRP & 4 & 4 & 2 & 1 \\
EIGRP & 1 & 1 & 2 & 1 \\
OSPF & 3 & 3 & 1 & 2 \\
\hline
\end{tabular}

Table 2: Performance of RPs with link failure

\begin{tabular}{llll}
\hline RP/Application & e-mail & Database & HTTP \\
\hline RIPv2 & 4 & 4 & 4 \\
IGRP & 3 & 3 & 3 \\
EIGRP & 1 & 2 & 1 \\
OSPF & 2 & 1 & 2 \\
\hline
\end{tabular}

Analyzing the results in Table 1 and 2, we see that the most significant factors that affecting the performance of routing protocols are usage of resources and the convergence time.

With normal traffic (without failure) EIGRP outperform the other routing protocol. EIGRP uses medium resources, so, it performs well. While OSPF uses high resources to so, it takes more time. RIPv2 sometimes perform better than others because it uses low resource. The convergence time is fast in EIGRP and OSPF while it is slow in RIPv2. So, with failure traffic, EIGRP and OSPF perform better than RIPv2.

\section{CONCLUSION}

A comparative study of dynamic routing protocols over wired network is performed. Four dynamic routing protocols namely; RIPv2, IGRP, EIGRP and OSPF are investigated. Three different applications namely: e-mail, database and HTTP are utilized. Two scenarios are implemented. Traffic without failure and with failure. The results show that the most important factors that affecting the performance of these routing protocols are resource usage and convergence speed. EIGRP performs better with both scenarios. OSPF performs well in scenario 2 (with failure). RIPv2 sometimes performs well in scenario 1 (without failure). EIGRP is the best choice for a routing protocol on private networks because it offers the best balance between convergence speed, scalability and ease of management. OSPF is also a good choice in large topologies.

\section{REFERENCES}

Clark, M.P., 2003. Data Networks, IP and the Internet: Protocols, Design and Operation. John Wiley and Sons, Hoboken, New Jersey, USA., ISBN:9780470848562, Pages: 866.

Fitzgerald, J., A. Dennis and A. Durcikova, 2017. Business Data Communications and Networking. 13th Edn., John Wiley and Sons Publisher, Hoboken, New Jersey, USA., ISBN: 9781119368892, Pages: 400.

Krinpayorm, I. and S. Pattaramalai, 2012. Link recovery comparison between OSPF and EIGRP. Intl. Proc. Comput. Sci. Inf. Technol., 27: 192-197.

Meenakshi, A. Kaushik and Satvika, 2014. QoS parameter evaluation of UMTS under EIGRP, IGRP and RIP using OPNET. Proceedings of the International Conference on Communication and Signal Processing, April 3-5, 2014, IEEE, Melmaruvathur, India, ISBN:978-1-4799-3357-0, pp: 399-403.

Mohammad, Z., A. Abusukhon and M.A. Al-Maitah, 2017. A comparative performance analysis of route redistribution among three different routing protocols based on OPNET simulation. Intl. J. Comput. Networks Commun., 9: 39-55. 
Pethe, R.M. and S.R. Burnase, 2011. Technical era language of the networking-EIGRP. Intl. J. Eng. Sci. Technol., 3: 1-5.

Sharma, V., R. Narula and S. Khullar, 2012. Performance analysis of IEEE 802.3 using IGRP and EIGRP routing protocols. Intl. J. Comput. Appl., 44: 21-25.

Singh, J. and R. Mahajan, 2013. Simulation based comparitive study of RIPV2, OSPF and EIGRP. Intl. J. Adv. Res. Comput. Sci. Software Eng., 3: 1-4.
Verma, A. and N. Bhardwaj, 2016. A review on Routing Information Protocol (RIP) and Open Shortest Path First (OSPF) routing protocol. Intl. J. Future Gener. Commun. Networking, 9: 161-170.

Vetriselvan, V., P.R. Patil and M. Mahendran, 2014. Survey on the RIP, OSPF, EIGRP routing protocols. Intl. J. Comput. Sci. Inf. Technol., 5: 1058-1065.

Wandile, S., R. Zende, S. Sribhashyam, M.M. Kale and M.R. Jaiswal, 2017. Comparison of routing algorithms using riverbed modeler. Intl. J. Adv. Res. Comput. Commun. Eng., 6: 428-432. 